\title{
Metal hydroborates as solid-state electrolytes
}

\author{
R. Černý \\ DQMP, Laboratory of Crystallography, University of Geneva, Switzerland \\ Radovan.Cerny@unige.ch
}

This Interest in metal hydrides was initially driven by the potential to develop efficient and safe on-board hydrogen stores working close to ambient pressure and temperature. In search for hydrides with higher gravimetric storage capacity, the researchers concentrated on hydrides based on light atoms, among others on $\mathrm{Li}$ and $\mathrm{Na}$ salts containing hydroborate anions such as borohydride $\mathrm{BH}_{4}^{-}$or closohydroborate $\mathrm{B}_{12} \mathrm{H}_{12} 2^{2-}$ [1]. The hydrogen absorption-desorption cycling in complex hydrides still needs more chemical ideas due to relatively strong covalent bonding. Unexpectedly, the high mobility of alkali metal cations in some complex hydrides has opened the door for their application as battery materials, mainly as solid-state electrolytes (SSE).

Replacing the liquid electrolyte by SSE offers several advantages: $i$ ) a solid material is more thermally stable, thus enhancing the overall safety of the battery; ii) being less prone to the dendrite penetration, it enables the use of alkali metals as negative electrodes and iii) acting as physical layer between the two electrodes, it has a beneficial effect on the cell performance [2].

Among the different classes of SSE, the metal hydroborates have received particular interest, being soft, highly stable toward oxidation and exhibiting fast ion conductivity, enabled by an entropically-driven phase transition. Such transitions generally occur above room temperature $(r t)$, and it is therefore necessary to frustrate the anionic lattice, for example by anion mixing to bring the superionic regime down to $r t$ [3-6].

The hydrogen storage and mobility of the cations in light complex hydrides depends on the structural features, pathways available in the anion packing and on the anion thermal motion. While the latter requires important experimental and theoretical effort, the first two parameters can be easily quantified from crystal structures obtained by X-ray powder diffraction.

Examples of crystallography and crystal chemistry analyses of novel solid-state electrolytes as well as proof-of-concept Na-ion allsolid-state batteries will be shown.

[1] Paskevicius M. et al. Chem. Soc. Rev. 2017, 46, 1565

[2] Zeier W. \& Janek J. Nat. Energy 2016, 1, 16141

[3] Tang W.-S. et al. ACS Energy Lett. 2016, 1, 659

[4] Duchêne L. et al. Energy Environ. Sci. 2017, 10, 2609

[5] Murgia F. et al. Electrochem. Comm. 2019, 106, 106534

[6] Brighi M. et al. Cell Reports Phys. Sci. 2020, 1, 100217

Keywords: all-solid-state battery, solid-state electrolyte, metal hydroborate, powder diffraction 